\title{
Quality of life in myopia
}

Academic Department of Ophthalmology, Manchester Royal Eye Hospital, Manchester M13 9WH

K Rose

A Tullo

Department of

Optometry

R Harper

C Tromans

School of Nursing, Midwifery and Health Visiting, University of Manchester

C Waterman

The Eye Site, Manchester D Goldberg

Murray and Haggerty, Manchester

C Haggerty

Correspondence to: Dr K E Rose

atullo@

central.cmht.nwest.nhs.uk

Accepted 3 May 2000

Karen Rose, Robert Harper, Cindy Tromans, Christine Waterman, David Goldberg, Clare Haggerty, Andrew Tullo

\begin{abstract}
Background-The safety and predictability of refractive surgery for all degrees of myopia is now becoming established. It is therefore appropriate to evaluate whether there is a patient driven demand for such treatments and, if so, to establish guidelines for its provision within the National Health Service (NHS).
\end{abstract}

Methods-A comparative study was designed to assess the effect of degree of myopia on quality of life ("high" $(n=30)$ $-10.00 \mathrm{D}$, worse eye; "moderate" $(n=40)$ -4.00 to $-9.75 \mathrm{D}$, worse eye; "low" $(n=42)$ $<-4.00 \mathrm{D}$, worse eye) compared with a group of patients with keratoconus ( $n=$ 30) treated by optical correction. Data collection included binocular $\log$ MAR visual acuity, Pelli-Robson low contrast letter sensitivity, questionnaires to assess subjective visual function (VF-14) and effect on quality of life (VQOL), and semistructured interviews.

Results-There were no significant differences in any of the measures between patients with a high degree of myopia and those with keratoconus, or between those with a low and those with a moderate degree of myopia. However, those with a high degree of myopia had highly significantly poorer $\log M A R, V F-14$, and VQOL scores than those with low and moderate myopia $(p<0.001)$. Interview data supported these findings with patients with a high degree of myopia and those with keratoconus reporting that psychological, cosmetic, practical, and financial factors affected their quality of life.

Conclusion-Compared with low and moderate myopia, patients with a high degree of myopia experience impaired quality of life similar to that of patients with keratoconus. Criteria should therefore be identified to enable those in sufficient need to obtain refractive surgical treatment under the NHS.

(Br f Ophthalmol 2000;84:1031-1034)

Myopia affects $25 \%$ of the population in western industrialised societies and has a potentially negative effect on self-esteem, career choice, and ocular health. ${ }^{12}$ Despite the fact that the experience of being myopic appears to include a psychosocial component, the authors are unaware of published studies which indicate whether or not subjects with myopia feel that they have a significantly impaired quality of life. ${ }^{3}$

At present much of the interest in myopia centres around the nature versus nurture debate $^{14}$ and evaluation of new treatments such as photorefractive keratectomy (PRK), laser in situ keratomileusis (LASIK), and phakic lens implant. Although the evidence as to whether LASIK should replace PRK for any particular range of myopia is still not conclusive, ${ }^{5}$ most patients who present for laser treatment are seemingly satisfied with the outcome, ${ }^{6-8}$ including patients with higher degrees of myopia who undergo LASIK. ${ }^{9-11}$

Patients who undergo such treatments are essentially a self selected group and therefore do not provide representative information on what it is like to live with myopia of different degrees or whether there is a patient driven need for provision of such treatments.

A study was therefore designed with the following objectives: (1) to assess the effect of myopia and its degree on quality of life in spectacle and contact lens wearers; (2) to compare the quality of life in patients with myopia with that in patients with keratoconus (a group of patients who suffer refractive change warranting ophthalmic management, including surgery, under the NHS); and (3) to inform future research into, and implementation of, alternative treatments for myopia.

\section{Patients and methods}

A comparative design was adopted consisting of the following four groups of adult patients aged between 18 and 65 :

Group 1: High degree of myopia (refractive error $\geqslant-10.00 \mathrm{D}$, worse eye; $\geqslant-8.00 \mathrm{D}$, better eye; $<2.00 \mathrm{D}$ anisometropia);

Group 2: Moderate degree of myopia $(-4.00 \mathrm{D}$ to $-9.75 \mathrm{D}$, worse eye; $\leqslant 2.00 \mathrm{D}$ anisometropia);

Group 3: Low degree of myopia $(-1.50 \mathrm{D}$ to $-3.75 \mathrm{D}$, worse eye; at least $-1.00 \mathrm{D}$, better eye; $\leqslant 2.00 \mathrm{D}$ anisometropia);

Group 4: Patients with bilateral keratoconus requiring correction by contact or spectacle lenses.

Data collection took place between February and October 1998. Patients in groups 1, 2, and 3 were recruited from five optometric practices covering areas of differing socioeconomic affluence in the Manchester area. Patients were eligible for inclusion if they had undergone a routine eye test in the previous 2 years, met a minimum best corrected visual acuity of $6 / 12$ in the worse eye, and had not put themselves forward for laser treatment; those meeting the inclusion criteria were invited by letter to participate. In group 4, 32 consecutive patients attending the optometry department at Manchester Royal Eye Hospital (MREH) who met a minimum best corrected visual acuity of $6 / 18$ in the worse eye and were deemed "stable" in 
that refitting of lenses had not been required for at least 6 months were invited to take part. In all groups patients with a history of ocular comorbidities, previous ocular surgery, or diabetes were excluded. Approval was obtained from the local research ethics committee.

Patients with myopia were assessed at their optometrist's practice and those with keratoconus at MREH. All the patients wore their habitual spectacle or contact lens correction for assessment so that the data might better reflect their "real world" visual experience.

The patients were assessed by a single researcher as follows: binocular logMAR visual acuity, Pelli-Robson low contrast letter chart (both charts illuminated to a minimum of $120 \mathrm{~cd} / \mathrm{m}^{2}$ ), questionnaires evaluating subjective visual function $(\mathrm{VF}-14)^{12}$ and vision related quality of life (VQOL), ${ }^{13}$ semistructured interviews, and estimation of cost of prescriptions to patients.

The scoring ranges for the quantitative measures were as follows:

(1) LogMAR: a score of 0.0 is equivalent to $6 / 6$ vision, with minus scores indicating better vision and plus scores indicating poorer vision;

(2) Pelli-Robson low contrast letter chart: scores range from 0.00 , rising in intervals of 0.15 to 2.25 , higher scores indicating more acute perception;

(3) VF-14: score range $0-100$. The lower the score, the more difficulty the respondent has performing activities tested;

(4) VQOL: score range 0-5. High scores indicate poorer vision related quality of life.

The interviews consisted of a structured component where patients were asked about

Table 1 Age, sex, type of lens correction

\begin{tabular}{|c|c|c|c|c|c|c|c|}
\hline & \multicolumn{3}{|l|}{ Age } & \multicolumn{2}{|c|}{ Sex } & \multicolumn{2}{|l|}{ Lens } \\
\hline & Mean & Median & Range & $M$ & $F$ & Spectacle & Contact \\
\hline Group $1(n=30)$ & 45 & 46 & $21-64$ & 8 & 22 & 19 & 11 \\
\hline Group $2(n=40)$ & 42 & 43 & $18-65$ & 12 & 28 & 26 & 14 \\
\hline Group $3(n=42)$ & 41 & 43 & $18-60$ & 12 & 30 & 26 & 16 \\
\hline Group $4(n=30)$ & 35 & 32 & $24-60$ & 22 & 8 & 3 & 27 \\
\hline
\end{tabular}

Table 2 Mean and median scores by group for $\log M A R$, low contrast letter sensitivity, $V F-14$, and $V Q O L$

\begin{tabular}{lcccc}
\hline & Group 1 & Group 2 & Group 3 & Group 4 \\
\hline logMAR & 0.12 & -0.04 & -0.08 & 0.05 \\
$\quad$ Mean & 0.12 & -0.03 & -0.10 & 0.02 \\
$\quad$ Median & 1.77 & 1.89 & 1.89 & 1.81 \\
Contrast (range 0-2.25) & 1.80 & 1.95 & 1.95 & 1.80 \\
$\quad$ Mean & & & & \\
$\quad$ Median & 83.90 & 94.70 & 97.02 & 89.84 \\
VF-14 (range 0-100) & 89.25 & 95.05 & 98.10 & 93.05 \\
$\quad$ Mean & 1.31 & 0.44 & 0.42 & 0.79 \\
$\quad$ Median & 1.20 & 0.25 & 0.20 & 0.60 \\
$\quad$ VQL (range 0-5) & & &
\end{tabular}

Table 3 Between groups comparison of statistical significance of $\log M A R$, low contrast letter sensitivity, $V F-14$, and $V Q O L$

\begin{tabular}{lrrrrrr}
\hline & Group & & & & \\
\cline { 2 - 7 } & \multicolumn{1}{c}{$1 v 2$} & $1 v 3$ & $1 v 4$ & $2 v 3$ & $2 v 4$ & $3 v 4$ \\
\hline $\operatorname{logMAR}(\mathrm{p}<0.05)$ & $<0.001$ & $<0.001$ & 0.189 & 0.636 & 0.001 & 0.028 \\
Contrast $(\mathrm{p}<0.01)$ & 0.013 & 0.010 & 0.650 & 0.814 & 0.015 & 0.010 \\
VF-14 (p<0.01) & $<0.001$ & $<0.001$ & 0.087 & 0.013 & 0.088 & $<0.001$ \\
VQOL $(\mathrm{p}<0.01)$ & $<0.001$ & $<0.001$ & 0.037 & 0.222 & 0.025 & 0.005 \\
\hline
\end{tabular}

their preferred form of optical correction, its cost, and about their knowledge of and interest in laser treatment of short sightedness. To complement the quantitative data collection methods ${ }^{14}$ patients were also asked the open question: "Do you feel that being short sighted has affected your work, your personal life or your social life in any way either now or in the past?"

\section{Results}

SAMPLE

One hundred and twelve patients with myopia who met the inclusion criteria were interviewed (28\% response rate). These were divided into groups by degree of refractive error in the worse eye, as described above. Table 1 shows the numbers, age, sex, and mode of lens correction (spectacles or contact lenses) for each of the groups.

STATISTICAL RESULTS

The mean scores for binocular logMAR visual acuity, binocular Pelli-Robson low contrast letter sensitivity, VF-14, and VQOL are shown in Table 2. Statistical analysis of these variables showed that only the variable $\log M A R$ was normally distributed. A one way ANOVA (significant at the 0.05 level, 95\% confidence interval) was used to test the differences in visual acuity between the four groups (Table 3). Patients in group 1 (high myopia) had highly significantly poorer binocular visual acuity than those with both low and moderate myopia $(\mathrm{p}<0.001)$. Patients in group 4 (keratoconus) had significantly poorer acuity than those with low $(\mathrm{p}=0.001)$ and moderate $(p=0.028)$ myopia. There was no significant difference in visual acuity between those with a high degree of myopia and those with keratoconus $(p=0.189)$, or between those with low and those with moderate myopia $(\mathrm{p}=0.636)$.

All other variables were not normally distributed. Mann Whitney tests were employed at a reduced level of significance $(p<0.01)$ to reduce the likelihood of making a type I error. The following results are all presented at this level (Table 3).

There were highly significant differences $(p<0.001)$ between patients in group 1 (high myopia) and those in group 3 (low myopia) with respect to subjective visual function (VF14) and vision related quality of life (VQOL), with those with a high degree of myopia being disadvantaged on both measures. Low contrast letter sensitivity was of borderline significance $(p=0.01)$, patients with a high degree of myopia scoring less well than those with a low level of myopia.

Patients with a high degree of myopia also fared worse than those with a moderate degree of myopia (group 2) with significantly poorer VF-14 and VQOL scores $(\mathrm{p}<0.001)$ and a contrast sensitivity score again approaching significance $(p=0.013)$. There were no significant differences on any of the measures between patients in group 1 (high degree of myopia) and those in group 4 (keratoconus).

Compared with patients in group 3 (low myopia), patients with keratoconus had signifi- 
cantly poorer VF-14 scores $(\mathrm{p}<0.001)$, a significantly poorer VQOL score $(\mathrm{p}=0.005)$, and borderline lower contrast sensitivity $(\mathrm{p}=0.01)$. However, compared with group 2 (moderate myopia), keratoconus patients showed no significant differences on any of the measures tested, although contrast sensitivity was reduced to a level approaching significance $(p=0.015)$. There were no statistically significant differences between patients in groups 2 and 3 (moderate and low myopia) on any of the variables.

INTERVIEW FINDINGS

All patients took part in interviews. These consisted of a structured part where patients were asked their preferred method of optical correction (Table 1) and how much this had cost (Table 4). It was found that patients with a high degree of myopia spent more money on spectacles than those with low or moderate myopia, even taking into account that they were entitled to vouchers to help with the cost of complex lenses. All groups were comparable in the amounts they spent on contact lenses and solutions, although it should be noted that patients with keratoconus tended to have to buy lenses more frequently than those with myopia because the fitting changed more rapidly as a result of disease progression.

All the patients with myopia were also asked whether they had heard of laser treatment for short sightedness and, if so, whether they would ever consider it themselves (Table 5). At least $90 \%$ knew that laser treatments existed, more in the groups with high and moderate degrees of myopia. These two groups of patients also expressed more interest in having the treatment, although many of those who were interested expressed reservations on the long term effects. In addition, the cost of such treatment was seen as prohibitive.

The unstructured part of the interviews was analysed qualitatively. ${ }^{15}$ Patients with a low degree of myopia were the most likely to make extreme statements about their short sight, while those with a moderate degree of myopia tended to play down the effect of their vision on daily functioning. Patients with a high degree of myopia and those with keratoconus were the most likely to cite concrete instances of how their eyesight adversely affected their lives. Examples fell into the following catego-

Table 4 Average cost to patients per annum (by group) of purchasing habitual form of optical correction

\begin{tabular}{lll}
\hline & Spectacles & Contact lenses \\
\hline Group 1 & $£ 230$ & Lenses $£ 60$, solutions $£ 120$ \\
Group 2 & $£ 130$ & Lenses $£ 80$, solutions $£ 95$ \\
Group 3 & $£ 90$ & Lenses $£ 90$, solutions $£ 100$ \\
Group 4 & $£ 100$ & Lenses $£ 140$, solutions $£ 120$ \\
\hline
\end{tabular}

Table 5 Patients with myopia aware of or willing to consider laser treatment

\begin{tabular}{lll}
\hline & Aware & Consider \\
\hline Group 1 $(\mathrm{n}=30)$ & $29(97 \%)$ & $16(55 \%)$ \\
Group 2 $(\mathrm{n}=40)$ & $38(95 \%)$ & $24(60 \%)$ \\
Group 3 $(\mathrm{n}=42)$ & $38(90 \%)$ & $9(24 \%)$ \\
\hline
\end{tabular}

ries: psychological, cosmetic, practical, and financial.

Many patients with a high degree of myopia reported that their eyesight dominated their lives from an early age. In many this led to a lack of self confidence because of teasing and feelings of inadequacy; this, in turn, could lead to social isolation and difficulties forming relationships. In others it led to a determination to succeed at activities that did not require perfect distance vision.

The psychological effects of a high level of myopia were augmented by cosmetic and financial factors. Many commented on their dislike of wearing thick spectacle lenses which were felt to be unsightly and a social handicap. Contact lenses were seen to have revolutionised the situation for those who could wear them, and wearers of high index spectacle lenses also commented on the positive cosmetic and social effects of their lighter, thinner lenses. However, the high cost of the latter, especially for higher prescriptions, limited the numbers who were able to take advantage of this option. The type of spectacle frame capable of supporting strong, complex lenses further restricted choice for those with a high degree of myopia, and several of the older individuals commented that years of wearing heavy glasses had damaged the skin on the bridge of the nose, further reducing options of what they could wear.

Both patients with a high degree of myopia and those with keratoconus commented that central distance vision was not the only factor in their visual experience. Practical difficulties were experienced in relation to discomfort in wearing contact lenses (especially patients with keratoconus), and with respect to peripheral vision (spectacle wearing patients with a high degree of myopia). In these groups the extreme dependence on optical correction for any kind of normal functioning was a constant daily concern. Difficulties in participating in sports such as swimming, football, cricket, and tennis were also frequently cited.

\section{Discussion}

SIGNIFICANCE OF FINDINGS

The quantitative results of this study show that higher degrees of myopia have an adverse effect on quality of life that is comparable to that of patients with an eye disease such as keratoconus which is widely accepted to be visually disabling and warranting management, including surgery, under the NHS. The visual disability of patients with a high degree of myopia and its effect on quality of life is further shown when comparisons are made with other groups. Both patients with keratoconus and those with a high degree of myopia were disadvantaged compared with those with a low degree of myopia. However, compared with patients with moderate myopia, those with keratoconus had no significant differences in any of the outcome measures while those with a high degree of myopia reported significantly reduced quality of life on all measures with the exception of low contrast letter sensitivity, which was borderline. The interview data sup- 
port the case that higher degrees of myopia are visually, personally, and financially disabling. The fact that patients with a high or moderate degree of myopia were much more likely to consider laser treatment also indicates that many of those who are significantly myopic feel that their needs are not being adequately met by current management methods.

\section{LIMITATIONS OF STUDY}

The low response rate among patients with myopia invited to participate in the study may have resulted in a bias towards those who are more conscious of their health and its effect on quality of life. Despite the fact that there appeared to be little variation between responders and non-responders in terms of age or post code, when evaluating the findings of the study it must be accepted that participants were to some extent a self selected rather than a representative group.

In assessing the significance of the results, the wide range of refractive error in the groups with a moderate and high degree of myopia should be considered. In addition, there is no "clear water" between groups so that those at the "high" end of one group may have a very similar degree of refractive error to those at the "low" end of another group.

The predominance of men (2:1) in the keratoconus group was predictable. ${ }^{16}$ The predominance of women in the myopia group is less easy to explain, although it was the researcher's impression that women were more flexible in arranging appointments and that more women may therefore have participated simply for logistical reasons.

One aspect of the study which merits further investigation is the findings related to binocular $\log$ MAR visual acuity. Despite the fact that the Snellen visual acuity entry criterion was included to ensure that all participants had broadly comparable best corrected distance vision, patients with a high degree of myopia exhibited significantly poorer binocular logMAR scores. One interpretation could be that the reduced performance of this group in relation to other variables was therefore simply a result of poorer corrected vision. However, this is countered to some extent by the fact that, although those with a high degree of myopia recorded poorer $\log M A R$ visual acuity scores than other patients with myopia, their results were comparable to the keratoconus group who had a less stringent Snellen acuity entry criterion, indicating that monocular Snellen acuity and binocular logMAR acuity may not be inextricably linked, but rather provide complementary information about visual status. In addition, it should be remembered that, in the study, "real life" visual experience was the variable being measured rather than best possible achieved vision.

CONCLUSIONS

Research is needed to replicate and refine this study to elaborate the needs of this group of patients. In particular, studies are required which differentiate between the experiences of non-presbyopic and presbyopic patients with myopia.

The results of this study indicate that high myopia has an adverse effect on quality of life. If refractive surgery has reached an acceptable level of safety and predictability, then it can be argued that the time has come to define criteria which would facilitate treatment of patients with myopia according to need-that is, within the NHS.

\section{Note added at proof stage:}

The VQOL questionnaire is now referred to as the VCM1 (Frost NA, Sparrow JM, Durrant JS, et al. Development of a questionnaire for measurement of vision-related quality of life. Ophthalmic Epidemiol 1998;5:185-210).

1 Mutti DO, Zadnik K, Adams AJ. Myopia: the nature versus nurture debate goes on. Invest Ophthalmol Vis Sci 1996;37:952-7.

2 Rosenfield M, Gilmartin B. Preface. In: Rosenfield M, Gilmartin B, ed. Myopia and nearwork. Oxford: ButterworthHeinemann, 1998:ix.

3 Rose KE, Tullo AB. Myopia. [Letter]. Br f Ophthalmol 1998;82:1220

4 Flitcroft DI. Ophthalmologists should consider the causes of myopia and not simply treat its consequences. $\mathrm{Br} \mathcal{F O p h}$ of myopia and not simply

5 Azar DT, Farah SG. Laser in situ keratomileusis versus photorefractive keratectomy: an update on indicators and torefractive keratectomy: an update on indicators

6 Freitas C, Oliveiros BM, Marques E, et al. Effect of photorefractive keratectomy on visual functioning and quality of life. F Refract Surg 1995;11 (3 Suppl):S327-34.

7 Hamberg-Nystrom H, Tengroth B, Fagerholm P, et al. Patient satisfaction following photorefractive keratectomy for myopia. F Refract Surg 1995;11(3 Suppl):S335-6.

8 McGhee CNJ, Orr D, Kidd B, et al. Psychological aspects of excimer laser surgery for myopia: reasons for seeking treatment and patient satisfaction. Br f Ophthalmol 1996;80: 874-9.

9 Pallikaris IG, Siganos DS. Excimer laser in situ keratomileusis and photorefractive keratectomy for correction of high myopia. F Refract Corneal Surg 1994;10:498-510.

10 Marinho A, Pinto MC, Vaz F, et al. Lasik for high myopia: one year experience. Ophthalmic Surgical Lasers 1996;27(5 Suppl):S517-20

11 Maldonado-Bas A, Onnis R. Results of laser in situ keratomileusis in different degrees of myopia. Ophthalmology 1998;105:606-11.

12 Steinberg EP, Tielsch JM, Schein OD, et al. The VF-14: an index of functional impairment in patients with cataract. Arch Ophthalmol 1994;112:630-7.

13 Frost NA. VQOL eyesight questionnaire. University of Bristol: Department of Ophthalmology, 1996,

14 Holman HR. Qualitative enquiry in medical research. 7 Clin Epidemiol 1993;46:29-36.

15 Rose $\mathrm{KE}$, Webb C. Analyzing data: maintaining rigor in a qualitative study. Qualitative Health Res 1998;8:556-62

16 Tuft SJ, Moodaley LC, Gregory WM, et al. Prognostic factors for the progression of keratoconus. Ophthalmology 1994;101:439-47. 